\title{
A Rare case of Monoclonal Gammopathy of Renal Significance with Immunotactoid Glomerulopathy Responding to Bortezomib, Dexamethasone and Rituximab
}

Chaoyuan Kuang ${ }^{1}$, Kevin Quann ${ }^{1}$, Agnes Liman², Roy Frye ${ }^{2}$, Nawras Alshoubaki ${ }^{3}$, Mohan Ramkumar $^{3}$, and Andrew Liman ${ }^{2}$

${ }^{1}$ University of Pittsburgh Cancer Institute

${ }^{2}$ VA Pittsburgh Healthcare System

${ }^{3}$ University of Pittsburgh Medical Center

May 5, 2020

\begin{abstract}
We report a rare case of monoclonal gammopathy of renal significance with immunotactoid glomerulopathy and severe nephrotic syndrome. The patient was diagnosed with a B-cell IgM $x$ clone and her nephrotic syndrome is responding well to bortezomib, dexamethasone, and rituximab.
\end{abstract}

A Rare case of MGRS with Immunotactoid Glomerulopathy Responding to Bortezomib, Dexamethasone and Rituximab

Short title: MGRS with ITG Responding to BDR

Chaoyuan Kuang ${ }^{1,2}$, Kevin Quann ${ }^{1,2}$, Agnes K. Liman ${ }^{3}$, Roy Frye $^{3}$, Nawras Alshoubaki ${ }^{4}$, Mohan Ramkumar $^{4}$, Andrew D. Liman ${ }^{1}$

1. Hematology/Oncology section, VA Pittsburgh Healthcare System

2. UPMC Hillman Cancer Center

3. Pathology and Laboratory Medicine, VA Pittsburgh Healthcare System

4. Renal section, VA Pittsburgh Healthcare System

Corresponding author:

Chaoyuan Kuang

UPCI Cancer Pavilion, Room 463

5150 Centre Avenue

Pittsburgh, PA 15232

kuangc@upmc.edu

T: 617-308-1715

F: 412-648-6579 
The abstract of this manuscript was presented at the Association of VA Hematology/Oncology meeting in Minneapolis, MN (Federal Practitioner, Abstract \#59, page S35, Sept 2019. www.mdedge.com/fedprac/avaho).

Main text word count: 1194

Figure count: 2

Reference count: 9

\section{Key Clinical Message:}

We strongly recommend patients with MGUS and unexplained proteinuria receive a renal biopsy to diagnose MGRS, because treatment by clone directed therapy against sources of monoclonal proteins can improve renal function in MGRS.

\section{Introduction}

Monoclonal gammopathy of renal significance (MGRS) is a recently described disorder caused by pathologic monoclonal protein (M-protein) leading to renal disease but little hematologic disease burden ${ }^{1}$. Failure to treat patients with MGRS can lead to poor outcomes due to progression of MGRS to more advanced monoclonal gammopathies, or end stage renal disease ${ }^{2}$. We report a case of MGRS leading to nephrotic syndrome and immunotactoid glomerulopathy (ITG). We hypothesized that aggressive treatment of the patient's MGRS through B-cell depletion and proteasome inhibition would improve her glomerulopathy and clinical outcomes. She is currently responding well to treatment with bortezomib, dexamethasone, and rituximab.

\section{Case Description}

A 66-year-old woman presented to our hematology clinic in 2016 with mild granulocytopenia and anemia. Her history was notable for iron deficiency anemia, vitamin D deficiency and laparascopic hysterectomy, though she was otherwise healthy. Her workup revealed a serum M-protein identified as an IgM $x$ clone at $0.28 \mathrm{~g} / \mathrm{dL}$. Her serum free light chain ratio was elevated at 2.23 , with a serum free $x$ of $22.9 \mathrm{mg} / \mathrm{L}$. She was diagnosed with monoclonal gammopathy of uncertain significance and monitored. In 2018, two years after her initial hematology consultation, she presented to nephrology clinic with acute kidney injury, peripheral edema, and hypertension. She had nephrotic range proteinuria at $6 \mathrm{~g} / 24$ hours, hematuria, and her urine studies suggested paraproteinuria. Her serum complement C3 was low at $68 \mathrm{mg} / \mathrm{dL}$. She underwent renal biopsy in April of 2018 which demonstrated immunotactoid glomerulopathy with membranoproliferative glomerulonephritis pattern (Fig 1A and 1B). Immunofluorescence of her renal biopsy showed $x$ light chain deposition in mesangial and capillary loops, with heavy IgM (Fig 1C) and moderate C3 staining (not shown). Electron microscopy revealed numerous immunotactoid deposits beneath the glomerular basement membrane (Fig 1D). Thioflavin and Congo Red staining were both negative for amyloid. Her serum M-protein burden was unchanged, and her bone marrow biopsy was nondiagnostic. Her bone marrow aspirate flow cytometry did identify a small $(<5 \%) \mathrm{CD} 20^{+}, \mathrm{CD} 5^{-}, \mathrm{CD} 10^{-}, \mathrm{CD} 23^{-}$, B-cell population with $x$ light chain restriction.

She was re-classified with MGRS and treated with rituximab $375 \mathrm{mg} / \mathrm{m} 2$ weekly, for four weeks, given over two separate courses. She experienced stable serum M-protein and free light chain burden, improvement of creatinine clearance and hypertension. However, she continued to have nephrotic syndrome. Her urine albumin/creatinine ratio only showed mild improvement from $8166 \mathrm{mg} / \mathrm{g}$ in November of 2018 to 7670 $\mathrm{mg} / \mathrm{g}$ in June of 2019. Due to her persistent renal dysfunction, we chose to treat with a Waldenstrom's Macroglobulinemia-inspired regimen consisting of bortezomib, dexamethasone, and rituximab, for a total of 5 cycles $^{3}$. On this regimen, her serum IgM, $x$ light chain, and creatinine have all normalized, while her urine albumin/creatinine ratio has improved dramatically to $1307 \mathrm{mg} / \mathrm{g}$ at time of this publication (Fig 2).

\section{Methods}

This report is a retrospective chart review of one patient with MGRS and immunotactoid glomerulopathy. 
The patient reviewed this manuscript and provided written informed consent for the review of her medical records and publication of the results, per the Declaration of Helsinki.

Bortezomib, dexamethasone, and rituximab $(\mathrm{BDR})^{3}$ :

Cycle 1: bortezomib $1.3 \mathrm{mg} / \mathrm{m} 2$ IV on days $1,4,8$, and 11; 21-day cycle.

Cycles 2-5: bortezomib $1.6 \mathrm{mg} / \mathrm{m} 2 \mathrm{IV}$ on days $1,8,15$, and 22; 35-day cycle.

Cycles 2 and 5 only: dexamethasone $40 \mathrm{mg}$ PO on days $1,8,15$, and 22 ; rituximab $375 \mathrm{mg} / \mathrm{m} 2 \mathrm{IV}$ on days $1,8,15$, and 22; 35-day cycle.

Contact corresponding author for data acquisition and microscopy details.

\section{Results and discussion}

MGRS is a new diagnostic classification encompassing patients who meet criteria for MGUS and otherwise have only renal disease caused by monoclonal gammopathy rather than clonal proliferation ${ }^{1,2}$. The spectrum of renal pathologies that fall under MGRS is evolving and are largely due to deposition of monoclonal Ig fragment in renal glomeruli ${ }^{4}$. Immunotactoid glomerulopathy (ITG) is defined by the pathognomonic finding of organized microtubular deposits with diameters of 10 to $60 \mathrm{~nm}^{4,5}$, as observed in our patient. ITG is frequently caused by IgG paraproteins and associated with CLL clones ${ }^{5,6}$. Our patient's workup revealed a CD5-, CD23-, B-cell clone, which precludes a CLL-related pre-malignancy. Further, IgM $x$ light chain was found to be the unifying lesion in our patient's studies, making her case even more unusual.

Clinicians currently have no firm guidelines for treatment of MGRS or suspected MGRS. The hematologic outcomes of this disease entity were only recently described. A large retrospective analysis by Steiner and colleagues found decreased PFS and OS in pathologically proven MGRS patients compared to MGUS patients ${ }^{2}$. Despite an imbalanced cohort number due to the rareness of MGRS cases, the authors clearly demonstrated that MGRS patient have increased risk of progression to more advanced monoclonal gammopathies such as multiple myeloma or smoldering myeloma. They also found MGRS patients to have a higher risk of progression to end stage renal disease (ESRD) as compared to MGUS patients. This observation is in line with older data from Heilman and colleagues showing that $82 \%$ of MGUS with light chain deposition patients progressed to (ESRD) despite therapy ${ }^{7}$. Thus, MGRS likely represents a distinct entity from MGUS with higher rates of both hematologic and renal disease progression. Under-recognition of the increased risk of progression of MGRS as compared to MGUS can lead to undertreatment and even misclassification. Our patient presented with MGRS, ITG, and classic signs of nephrotic syndrome. Based on currently available literature, she is at increased risk for progression to myeloma or ESRD as compared to MGUS patients. Given her minimal burden of comorbidities, we determined that it was in her best interest to treat her disease aggressively to prevent these outcomes.

Very little prospective data are available to guide treatment for MGRS. Expert opinion currently recommends a clone-directed approach ${ }^{1,5,8}$. Gumber and colleagues reported in a recent case series over a dozen MGRS patients treated by this approach ${ }^{9}$. Notably, nearly all of their patients had an IgG paraprotein or renal deposit identified, and most had no clonal involvement by bone marrow biopsy. Their treatment outcomes were encouraging, with most patients demonstrating at least partial response to rituximab-based treatment regimens. We discussed upfront aggressive treatment with our patient and opted for rituximab monotherapy first in an attempt to avoid more toxic therapies. Due to the minimal renal response to rituximab, marked by persistent massive proteinuria, we proceeded with a bortezomib-based regimen. We had also discussed employing a cyclophosphamide regimen given the patient's IgM paraprotein and renal dysfunction ${ }^{8}$, however due to the patient's desire to avoid toxic therapies and take a step-wise approach, we chose bortezomib, dexamethasone, and rituximab first ${ }^{3}$. Currently available anti-plasma cell and anti-B-cell regimens would likely all have some activity against the broad spectrum of MGRS clones, and more studies are needed to define which regimens are best for each pathology.

In summary, we present a rare case of MGRS with ITG caused by $\operatorname{IgM} \varkappa$ paraprotein from a non-CLL 
B-cell clone. Our patient has achieved a very good partial response to bortezomib, dexamethasone, and rituximab, and will be monitored for continued improvement. We strongly recommend that patients with MGUS and unexplained nephrotic range proteinuria be referred to nephrology for renal biopsy. MGRS is an under-recognized disease, but aggressive management can likely delay hematologic and renal progression of disease, leading to better long-term outcomes for patients.

\section{Acknowledgements}

We would like to acknowledge the UPMC Department of Pathology for technical support in performing transmission electron microscopy.

\section{Authorship contributions}

C.K. and A.D.L. conceptualized this manuscript. C.K. collected the data, drafted and revised this manuscript. A.D.L., K.Q., A.K.L. and R.F. collected data and assisted in the writing. All authors were involved in revising this manuscript and approve of it in its final form.

\section{Disclosure of Conflicts of Interest}

The authors have no conflicts of interest to declare.

\section{References}

1. Leung, N. et al. Monoclonal gammopathy of renal significance: when MGUS is no longer undetermined or insignificant. Blood120 , 4292-4295 (2012).

2. Steiner, N. et al. Monoclonal gammopathy of renal significance (MGRS) increases the risk for progression to multiple myeloma: an observational study of 2935 MGUS patients. Oncotarget 9 , 2344-2356 (2018).

3. Dimopoulos, M. A. et al. Primary therapy of Waldenström macroglobulinemia (WM) with weekly bortezomib, low-dose dexamethasone, and rituximab (BDR): Long-term results of a phase 2 study of the European Myeloma Network (EMN). Blood 122, 3276-3282 (2013).

4. Bridoux, F. et al. Diagnosis of monoclonal gammopathy of renal significance. Kidney Int. 87, 698-711 (2015).

5. Fermand, J.-P. et al. How I treat monoclonal gammopathy of renal significance (MGRS). Blood 122 , 3583-90 (2013).

6. Nasr, S. H. et al. Immunotactoid glomerulopathy: clinicopathologic and proteomic study. Nephrol. Dial. Transplant $27,4137-46$ (2012).

7. Heilman, R. L., Velosa, J. A., Holley, K. E., Offord, K. P. \& Kyle, R. A. Long-term follow-up and response to chemotherapy in patients with light-chain deposition disease. Am. J. Kidney Dis. 20 , 34-41 (1992).

8. Sethi, S. \& Rajkumar, S. V. Monoclonal Gammopathy-Associated Proliferative Glomerulonephritis. Mayo Clin. Proc. 88, 1284-1293 (2013).

9. Gumber, R. et al. A clone-directed approach may improve diagnosis and treatment of proliferative glomerulonephritis with monoclonal immunoglobulin deposits. Kidney Int. 94, 199-205 (2018).

\section{Figure Legends}

Figure 1. Microscopy of renal biopsy. A. PAS stain demonstrating mesangial expansion and mild segmental sclerosis (arrows). B. Jones stain demonstrating immune deposits (arrows) along glomerular capillaries which are negative for silver stain. C. Immunofluorescence stain for IgM showing 3+ staining on mesangial and capillary loops (arrows). D. Transmission electron microscopy showing numerous immunotactoid deposits composed of slightly curved, microtubular structures with mean diameter of $38.6 \mathrm{~nm}$, hollow cores, and parallel alignment beneath the glomerular basement membrane and mesangium.

Figure 2. Treatment course and lab parameters. BDR: bortezomib+dexamethasone+rituximab 


\section{Figure 1}
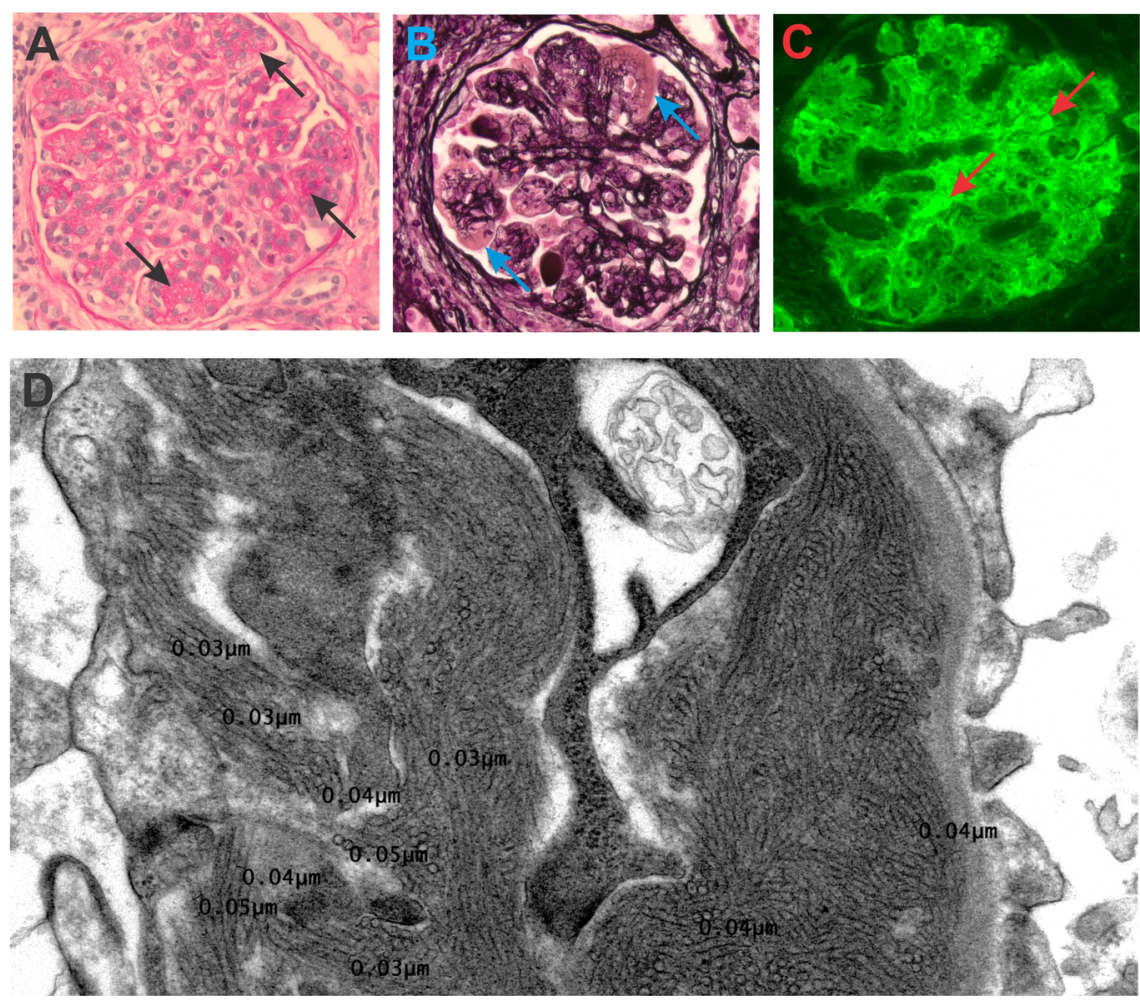

\section{Figure 2}

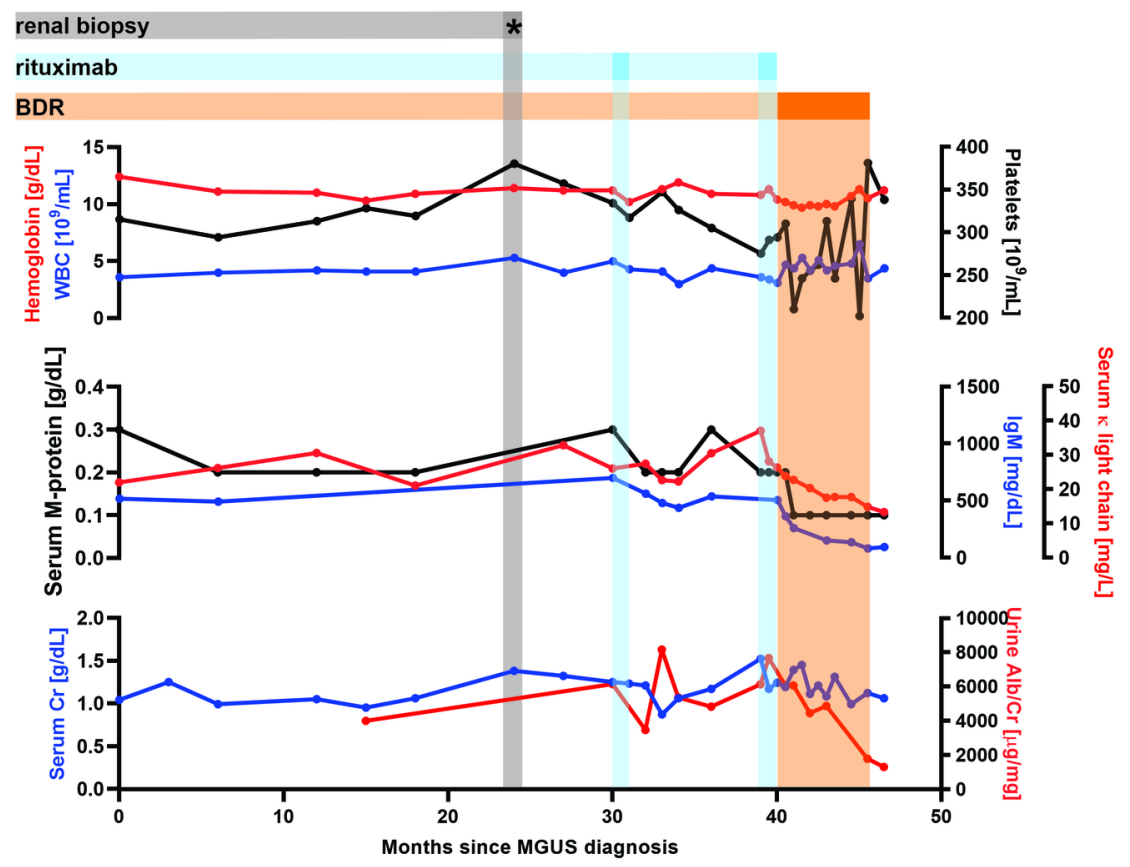

